\title{
BMJ Open Quality Integrating prevention and health promotion in a London prison
}

\author{
Adam John Finnie
}

To cite: Finnie AJ. Integrating prevention and health promotion in a London prison.BMJ Open Quality 2018;7:e000097. doi:10.1136/ bmjoq-2017-000097

Received 25 April 2017 Revised 22 November 2017 Accepted 8 December 2017

\section{CrossMark}

Health Promoting Trust Office, Education Centre, Eastbourne District General Hospital, Eastbourne, UK

Correspondence to Adam John Finnie; adam.finnie@gmail.com

\begin{abstract}
Many people enter prison with poor health from a background of deprivation and with unhealthy lifestyle habits, yet spending time in prisons is often actively detrimental to health. There is therefore a clear value in providing high-quality health promotion services in prisons that are effective at reaching those who need support to improve their lifestyle. The health promotion service at HMP Brixton provides a health trainers clinic to address lifestyle issues but found that it was sometimes challenging to identify appropriate patients and that the service was inefficient as a result. Analysis of our referral sources suggested that taking steps to increase the proportion of referrals made during screening and other clinics might lead to more appropriate and engaged patients. In this study, we set out to use quality improvement methods to increase referral from these sources. This involved improvements to the processes involved in delivering National Health Service Health Checks that were the primary source of referrals for health trainers and by improving links between the health trainer team and other clinicians. The changes were successful in increasing referrals from these sources. This work was completed during a period of exceptional disruption in the prison service and is relevant to secure healthcare sites that aim to ensure prevention activities are efficient and targeted.
\end{abstract}

\section{BACKGROUND}

In recent years, there has been a growing call for integrated health behaviour change services in prisons. ${ }^{123}$ It has been known for some time that men entering prison tend to have more unhealthy lifestyles than the general population; this concern is becoming more urgent in light of the fact that prison population is ageing, ${ }^{4}$ and there is a growing body of evidence that imprisonment contributes to unhealthy lifestyles and may contribute to lifestyle-related health issues such as obesity. ${ }^{5}$ A review in 2013 found that around one-third of UK prisons have implemented some kind of health or wellbeing services, ${ }^{6}$ but these have usually either been peer led by other prisoners ${ }^{7}$ or focused around exercise on referral and delivered within the gym, ${ }^{8}$ whereas services delivered in prison by health promotion professionals and integrated into the healthcare team remain scarce. In April 2015, the healthcare team at HMP Brixton began work to implement new services aimed at promoting behaviour change towards healthier lifestyles among prisoners. Those prisoners who were referred for healthy lifestyle advice would receive six appointments with a health trainer in which they were given advice and motivational support to change lifestyle behaviours such as dietary choices, exercise motivation and smoking. There is considerable evidence that health trainers can be an effective service to support healthier lifestyles for a targeted population, ${ }^{9}$ and early efforts to implement health trainers in prisons have showed positive results. ${ }^{10}$ Although the HMP Brixton health trainers were performing well in the first year, there was an ongoing issue that some patients were repeatedly not attending or attending for inappropriate reasons, and it was noted that this often occurred shortly after a surge of new referrals was received during promotional activities. A key aim of health trainer services is to reduce health inequality by focusing on accessing hard-toreach people, and this is usually achieved by running promotional events and outreach activities in deprived areas. In the community, this method is often found to be challenging as the public may lack motivation to engage, ${ }^{11}$ but it is also effective in reaching the right people. When initially conducting such activities in HMP Brixton, it was not found to be difficult to engage the men and many would self-refer. However, informal feedback from the health trainer team was that many such patients appeared to be healthy individuals who had referred themselves with motivations such as boredom, a desire to spend time out of their cell or to visit the healthcare department in order seek medication or to discuss unrelated issues.

These effects were particularly problematic in 2015-2016 when many UK prisons including HMP Brixton were experiencing severe operational challenges ${ }^{12}$ such as staff shortages which, in turn, led to reduced movements 'lockdowns' and cancelled healthcare clinics. In this context, it became particularly important for healthcare 
activities such our service to operate efficiently and reach the right patients.

Our initial response to this problem was to attempt to target men on an individual basis whom we had identified as less healthy from their notes and approach them directly in their cells. However, this approach had not led to a good rate of uptake.

In the analysis of our data at the end of the first year, a possible alternative solution was found; we observed that those patients who were referred into health promotion during the course of an appointment with a healthcare worker had better rates of attendance and were more likely to complete their course of appointments. This is in keeping with recent research showing that older men with health issues are more influenced to attend weight loss services if referred by a health professional or as a result of a new diagnosis. ${ }^{13}$ We therefore concluded that it would be possible to improve attendance to the health trainer clinics by encouraging other clinicians to refer more patients. The health trainers team also took on primary responsibility for delivering NHS Health Checks in order to ensure that this new clinic would also refer into health trainers. NHS Health Checks is a national programme that offers screening to people aged 40-74 years in order to assess the risk of cardiovascular disease and which is now required to be offered by healthcare providers in prison. ${ }^{14}$ Signposting to lifestyle interventions such as health trainers is a key purpose of health checks.

\section{PROBLEM}

The performance of the health trainer service was being hindered by patients attending infrequently and for the wrong reasons. Preliminary efforts to address the problem had found that self-referrals were a likely cause, whereas patients referred from screening and clinicians often achieved better attendance outcomes. A new NHS Health Checks screening service that was set up around the time the quality improvement (QI) project began was hoped to provide a source of appropriate referrals. Initially this was not the case and very few referrals from health checks to health trainers were being made. Therefore, one aspect of the project was to identify why referrals from NHS Health Checks were slow and take steps to resolve the problems where possible. The second aspect of the problem was that referral from clinicians were also infrequent. We believed that some clinicians were missing opportunities to refer and might do so if given reminders or encouragement. We also suspected some clinicians were not convinced of the benefits of referring to this service and that better engagement was required in order to build partnerships and raise their awareness. A goal was set to increase the number of referrals made by healthcare professionals and during screening clinics by $50 \%$ from the baseline of 41 in 2015-2016 (10.25 per quarter) up to 16 or more per quarter in 2016-2017.

\section{BASELINE MEASUREMENT}

The project began in April 2016. The baseline measurement was the number of referrals from clinicians and screening during the year prior to the improvement work: from April 2015 to April 2016. During the first year, 266 patients were referred to the health trainer service. Of these, 225 were self-referrals, 17 were referred by nurses, 11 by doctors, 10 by mental health staff, 2 by pharmacy, 1 by operational staff and 20 were not recorded. In line with our hypothesis, these were divided into 'self-referrals' $225(78 \%)$ who referred themselves into the programme during promotional activities on the wings of the prison. Those who were referred by healthcare staff during clinic or screening activities were designated 'clinician referrals', of which there were $41(14 \%)$ in 2015-2016. We planned to collect data on referral sources at quarterly intervals. It was possible to break these data down by month for further analysis at the end of the project (see table 2).

The decision was made to target the process goal of referral source rather than a performance outcome due to the level of disruption in the prison at the time. The outcomes which we eventually aimed to improve such as attendance were often also affected by unexpected changes in the operational status of the prison (such as lockdown periods or mass movements of prisoners). Since it would not have been clear during the process if improvement works if changes had affected these outcomes, we chose to focus on the referral pathway which was within our control.

\section{DESIGN}

The decision to carry out QI work to improve the performance of the health promotion service was made by the health promotion lead in March 2016. This period was identified by the health promotion lead and healthcare management as an appropriate moment to carry out improvement work both in order to address the lessons of the first year and also to maximise the potential to integrate health checks and health trainers. All QI activities were carried out by the health promotion lead with support from the lead health trainer and ongoing oversight from the head of healthcare. At a later stage, a nurse became part of the QI team, and the pharmacist was closely consulted regarding all work on NHS Health Checks. The QI team updated the full healthcare team on their progress in each monthly staff meeting and consulted the two doctors at the outset and individually over the course of the project.

A process to review the impact of improvement work was originally designed to take place at quarterly intervals alongside quarterly performance reporting activities. However, for clarity, a number of actions carried out in late September of Q2 are reported as Improvement Cycle 2. Improvement Cycle 3 refers to activities initiated at the beginning of Q4: December. The overall project was planned to be reviewed after 1 year on 1 April 2017. 
The project took a two-pronged approach in increasing appropriate referrals:

1. To monitor the NHS Health Checks and address any issues that prevented onward referrals.

2. To promote referral by other clinicians through engagement work and reminders.

During this period, the source of referral for each patient was recorded by health trainers into a case load monitoring document. The data were double checked using reports extracted from the clinical system for information such as referral read codes and messages from clinicians.

\section{STRATEGY}

Improvement Cycle 1: promoting uptake of health checks Plan

The first possible cause of low referrals to health trainers was simply that very few health checks were being completed. In order to identify possible causes of this problem, a discussion was held within the health promotion team in which the process from recruitment to delivery was mapped, and a potential explanation emerged. Team members were finding that the process of recruitment was slow because the lists of eligible patients that were taken onto the wings for recruitment were often inadequate since they included prisoners who had been moved, people who had already had their appointment and those who were not interested.

Second, the records of some of the patients eligible for health checks were reviewed for further information. It was identified that some of them had very frequent healthcare appointments but that the opportunity to refer them for a health check was being missed because staff were not aware they were eligible.

Do

In order to address the inefficiencies in the NHS Health Checks recruitment process, a number of changes were made: first, in order to ensure the list was up to date, the eligibility report was run monthly rather quarterly as was advised by Public Health England, ${ }^{15}$ and those had attended already were removed from the list straight away (as opposed to waiting for it to update automatically). Second, those patients who verbally refused were recorded with a read code and were removed from the list when it was compiled. In order to address the issue of missed opportunities to refer clinicians offered to have an automatic alert added in System One, which would present a pop-up reminder that someone was eligible for a health check.

\section{Study}

After this work was carried out, the number of completed health check screenings did increase (Q1=12, Q2=21, Q3=31); however, this did not lead to a corresponding improvement in the primary goal of increasing referrals to health trainers.

\section{Act}

The aim was set to improve the appointment process in health checks to ensure referrals were made when patients attended and to engage clinicians as part of this activity.

Improvement Cycle 2: improving health check appointments Plan

In Cycle 2 we sought to address the lack of referrals from health checks to the health trainers' clinics. Staff delivering the health checks reported that because the process involved multiple measurements, this could distract from the need to promote referrals. Also they were experiencing technical difficulties producing Q-Risk scores during the appointment.

Extra guidance was sought from clinicians for possible improvements to the health checks service in order that they would be more likely to lead to lifestyle discussion. One general practitioner (GP) with a particular interest in cardiovascular health suggested that giving more information about cardiovascular risk might engage men more and suggested alternative risk scores and informational resources we could use.

Do

In order to encourage staff to make referrals, the referral page in the health checks clinical template was updated to include specific references to health trainers where it had previously referred to 'weight management'. An instructional document was produced that pointed out the most time efficient sequence in which to complete measurements and step-by-step guidance for the technical issue. Following the GP's suggestion, online risk calculators were also used as an alternative, and extra information on cardiovascular health were given during the process.

\section{Study}

Referrals from health checks to health trainers did increase in Q3 indicating the improvements had some benefits; it should be noted that referrals from the GPs also increased suggesting the joint work encouraged referrals. However, there was great variability month to month: all the referrals in Q3 were made in November and none in October or December. This was due to an unforeseen issue of severe operational staff shortages reducing the capacity to deliver non-urgent clinics such as health checks.

Act

In light of the fact that health checks could not be relied on as a regular source of referrals, the goal was set to focus efforts on increasing referrals from regular clinics provided by doctors and nurses.

\section{Improvement Cycle 3: promoting referral from other clinicians \\ Plan}

Previous research has shown the importance of bridging professional boundaries during implementation work and the support of a small number of champions tending 
to underpin success. ${ }^{16}$ In line with guidance on reviving stalled projects, ${ }^{17}$ we set out to gain the support of wellknown and respected staff and to involve them further in proposing solutions. A number of informal meetings were held with the GPs and the nursing team to discuss how patients appropriate for lifestyle support might be identified and referred from their clinics. One nurse took lead responsibility for health improvement and provided a number of suggestions focused around identifying appropriate patients at reception (when they enter the prison). The first aim was to increase referrals from nurses during the healthcare reception screening at the moment of arrival. Second, a GP who was responsible for seeing all patients with diabetes on arrival in the prison offered to work together to ensure they were all offered lifestyle support.

Do

In order to encourage more referrals from reception screening, a reminder was added to the reception clinical template instructing nurses to refer patients to the health trainers. The health promotion nurse followed up with the nursing team frequently to ensure everyone understood the process and benefits. The health promotion lead ran a monthly report on patients with diabetes from December onwards and conferred with the GP to ensure all were assessed and seen by health trainers if willing.

\section{Study}

Referrals from doctors and nurses increased substantially and were at their highest level in Q4.

Act

The health promotion lead and nurse set out to build on this improvement with a future plan to screen all new reception patients for lifestyle issues as an opt-out service.

\section{RESULTS}

Referrals from screening and clinicians into health trainers programme did increase significantly over the improvement period from a baseline average of 10 per quarter to an average of 31.25 per quarter over the year studied (see table 1). There were consistent improvements in each consecutive cycle, although there was wide variability month on month and between sources of referral $(\mathrm{M}=9 \mathrm{SD}=9.9)$. It is clear from the run chart that at certain points in the year all types of referral dropped simultaneously (May, October and December; see figure 1). It was noted that all of these months corresponded with high levels of operational disruption in the prison. Following improvements to the health checks process in cycle 1 and 2, there were higher levels of referrals in the months not affected by disruption in November and January (November: $n=8$, January: $n=6$, see table 2). Referrals from clinicians also declined in the disrupted periods in October and December. Referrals from doctors and other clinicians increased substantially over the course of the study period, the total of 93 such referrals over the year represents a $126 \%$ increase on the previous year substantially in excess of our goal of a $50 \%$ increase. Referrals from other clinicians increased somewhat in Q2 $(n=20)$ and Q3 $(n=32)$, which may be attributable to working in partnership with other clinicians on health improvement pathway and generally raising awareness. In Cycle 3, efforts were specifically made to remind other clinicians to refer, and there was a large increase subsequently in $\mathrm{Q} 4(\mathrm{n}=57)$.

\section{LESSONS AND LIMITATIONS}

Referrals into the Health Trainers service did increase as intended however this improvement study did not control for possible confounding variables and therefore it cannot be known whether changes in performance occurred as a result of planned QI activities or whether they were an indirect result of another factor. The improvements seen may have resulted from chance, general increased effort from staff involved or improvements going on in the organisation unrelated to the QI actions. Normal prison operations may also have had a confounding effect on this data because for example when large numbers of men arrived at one time many new cases and referrals might result. In some cases, it was difficult to define what the original source of referral for a patient was; for example, some patients were referred twice in a short period of time. In these cases, the initial source was used. It is also not always clear whether a referral was a 'self-referral'; in

Table 1 Referral sources baseline and quarterly

\begin{tabular}{|c|c|c|c|c|c|c|c|c|}
\hline Referral sources & $\begin{array}{l}\text { April 2015- } \\
\text { April } 2016 \\
\text { Total } \\
\end{array}$ & $\begin{array}{l}\text { Average per } \\
\text { quarter 2015-2016 }\end{array}$ & $\begin{array}{l}2016 \\
\text { Q1 }\end{array}$ & $\begin{array}{l}2016 \\
\text { Q2 }\end{array}$ & $\begin{array}{l}2016 \\
\text { Q3 }\end{array}$ & $\begin{array}{l}2017 \\
\text { Q4 }\end{array}$ & $\begin{array}{l}\text { 2016-2017 } \\
\text { (FY) Total }\end{array}$ & $\begin{array}{l}\text { Average per quarter } \\
2016-2017\end{array}$ \\
\hline Doctor & 11 & 2.75 & 5 & 4 & 10 & 25 & 44 & 11 \\
\hline Healthcare professional & 30 & 7.5 & 4 & 12 & 8 & 25 & 49 & 12.25 \\
\hline NHS Health Checks & 0 & 0 & 7 & 4 & 14 & 4 & 29 & 7.25 \\
\hline Total pro-ref & 41 & 10.25 & 16 & 20 & 32 & 57 & 125 & 31.25 \\
\hline Self-referrals & 225 & 56.25 & 39 & 71 & 18 & 27 & 155 & 38.75 \\
\hline Total referrals & 266 & 66.5 & 55 & 91 & 50 & 84 & 280 & 70 \\
\hline
\end{tabular}

NHS, National Health Service. 


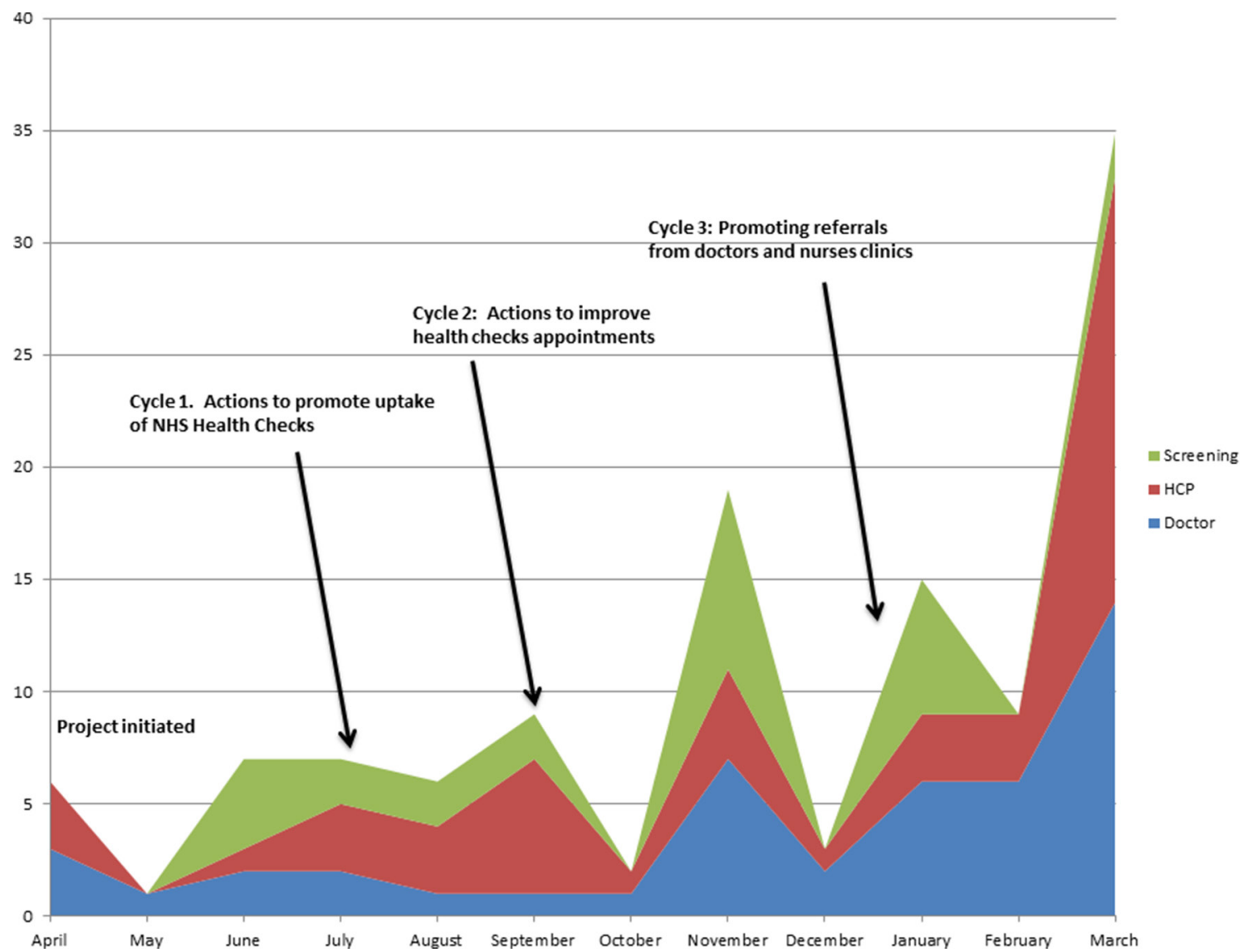

Figure 1 Annotated run chart of referrals sources by month, April 2016- April 2017. HCP, healthcare practitioner; NHS, National Health Service; PDSA, Plan, Do, Study and Act.

this study, all referrals that were received from clinicians were counted as 'clinician referral' on the basis that the clinician was able to triage and advise when the referral was made; however, it should be noted that in some cases, these referrals would have been directly requested by the patient with minimal input from the clinician. It cannot be shown with certainty whether increasing clinician referrals resulted in better engagement without comparing referral source to outcomes for individual patients that is beyond the ethical scope of this study. It should be noted that in each cycle a number of different actions were undertaken towards a particular improvement aim and therefore it is not possible to say precisely which activity in each cycle may have influenced the outcome measure. It would have been preferable to have implemented and studied smaller changes individually over a longer period but this was not feasible. Although this project was partly an effort to overcome operational challenges within the prison, it was nonetheless affected by them. During some periods activities were so severely disrupted that doctors and nurses who might have referred were not seeing patients, this was particularly the case in cycle 3 from October to December. A lesson from this may be that improvements that require changes to working patterns are less likely to succeed in prisons when the institution is generally under strain. Some of the greatest obstacles in conducting health promotion in prison were not able to address in this project. These have to do with the allocation of resources both in terms of people and space; for example, the nurse involved in health promotion was often reallocated to other duties at short notice, and the capacity to deliver health promotion clinics was often limited by the availability of rooms. These

Table 2 Referral sources by month

\begin{tabular}{llllllllllll}
\hline & April & May & June & July & August & September & October November & December January February March \\
\hline Doctor & 3 & 1 & 2 & 2 & 1 & 1 & 1 & 7 & 2 & 6 & 6 \\
Healthcare & & & & & & & & & & & \\
practitioner & 3 & 0 & 1 & 3 & 3 & 6 & 1 & 4 & 1 & 3 & 3 \\
Health check & 0 & 0 & 4 & 2 & 2 & 2 & 0 & 8 & 0 & 6 & 0 \\
\hline
\end{tabular}


problems highlight the fact that health promotion is sometimes viewed as a low priority in prison, and this may threaten the long-term sustainability of the work described here.

\section{CONCLUSION}

The three cycles of improvement emphasised increasing uptake of health checks, increasing referrals from health checks to the interventions and increasing referrals from other clinicians. In all of these three stages, it was found that adapting the clinical templates to include reminders, alerts and instructions to refer was effective. This indicates that integrating reminders to refer to prevention within existing IT systems works well in this context possibly because men in prison tend to visit healthcare frequently, and there a many opportunities for these reminders to be acted on. The fact that it was possible to increase referrals with reminders also supported our view that men in prison with a need for lifestyle support were more likely to accept in the context of an appointment possibly because of the privacy or existing rapport with the clinician.

Several of the lessons from this work highlight the importance of creating systems that are well adapted to the constant population churn of prisons in which patients arrive, move location and move out much more frequently than in normal community. The decision to run and update the health check eligibility lists and diabetic patient list more frequently both supported referrals into the health trainer intervention. Similarly, steps taken to increase referrals from reception screening in Cycle 3 were driven by the fact that monitoring new arrivals is often the best way to identify unmet needs in prison.

The overall aim to link health promotion activities more closely with other clinicians in order to promote referrals was successful. We found that referrals from some GPs and pharmacists increased considerably after we had conversations with them individually to explain the purpose of the health trainers or seek their guidance and increased further when they began to see outcomes and that were contributing to targets such as CQUINS (Commissioning for Quality and Innovation). Referrals from nurses increased when the service was regularly discussed in meetings and increased further when automatic reminders were added to their clinics. This might suggest that doctors need to see tangible evidence of positive patient outcomes before they refer to health trainers, whereas nurses need encouragement and reminders to make referrals part of their routine. In both cases, our experiences echoed those cited a recent Royal Society for Public Health report on health trainers, ${ }^{18}$ which found there were considerable difficulties for Health Trainers to overcome in the fact that other clinicians often do not understand what they do. There is a parallel between what we found in our effort to increase clinician referrals and our attempts to promote uptake of screening in that we needed to persuade people (either prisoner or clinician) that the service was credible and useful. This perhaps emphasises the fact that because health promotion services lack widely recognised job titles and a well understood clinical purpose that it is particularly important to design services in a way that builds relationships. In the same sense that we found prisoners needed to be gradually introduced to health improvement through a pathway from screening to intervention, it was also the case that clinicians gradually became more willing to refer to these interventions as they became familiar with the staff delivering them and understood the benefits. In light of these findings, we have begun work to create a second reception that includes a cardiovascular screening and well-being assessments that is given to all prisoners as an opt-out service.

Acknowledgements The author would like to thank Eunice Adu-Appiah, lead health trainer; Morgan Owen, head of healthcare; Dr Nial Aye Muang, general practitioner; Dr Nandana Jayaram, general practitioner; Magdalena Henzel, nurse; and Sheila Louis, pharmacist.

Competing interests None declared.

Provenance and peer review Not commissioned; externally peer reviewed.

Open Access This is an Open Access article distributed in accordance with the Creative Commons Attribution Non Commercial (CC BY-NC 4.0) license, which permits others to distribute, remix, adapt, build upon this work non-commercially, and license their derivative works on different terms, provided the original work is properly cited and the use is non-commercial. See: http://creativecommons.org/ licenses/by-nc/4.0/

(c) Published by the BMJ Publishing Group Limited. For permission to use (where not already granted under a licence) please go to http://www.bmj.com/company/ products-services/rights-and-licensing/

\section{REFERENCES}

1. Møller L, Stöver H, Jürgens R, et al. Health in prisons. 1st edn. Copenhagen: World Health Organization, 2007.

2. Bradshaw R, Pordes BA, Trippier $\mathrm{H}$, et al. The health of prisoners: summary of NICE guidance. BMJ 2017;356:j1378.

3. Howse K. Growing old in prison: a scoping study on older prisoners. London: The Prison Reform Trust, 2011.

4. Senior J, Forsyth K, Walsh E, et al. Health and social care services for older male adults in prison: the identification of current service provision and piloting of an assessment and care planning model. Health Services and Delivery Research 2013;1:1-138.

5. Gates ML, Bradford RK. The impact of incarceration on obesity: are prisoners with chronic diseases becoming overweight and obese during their confinement? J Obes 2015;2015:1-7.

6. Lewis $\mathrm{G}$. The benefit of sport and physical education for young men in prison: An exploration of policy and practice in England and Wales. Prison Service Journal 2013:3-11.

7. South J, Bagnall A-M, Hulme C, et al. A systematic review of the effectiveness and cost-effectiveness of peer-based interventions to maintain and improve offender health in prison settings. Health Services and Delivery Research 2014;2:1-218.

8. Lyttle G. Sheppey cluster RSPH Newsletter report, 2010.

9. South J, White J, Branney P, et al. Public health skills for a lay workforce: findings on skills and attributes from a qualitative study of lay health worker roles. Public Health 2013;127:419-26.

10. Centre for Clinical and academic workforce innovation. A guide to implementing the new futures health trainer role across the criminal justice system. University of Lincoln: Lincoln, 2007.

11. Mathers J, Taylor R, Parry J. Formulation and implementation of evidence-based public health policy in political environments: the health trainer service initiative in England. The Lancet 2017 http:// www.thelancet.com/ abstracts/public-health-scienceinthe- uk (accessed 10 Mar 2016)

12. HM Inspectorate of Prisons. HM chief inspector of prisons for England and Wales annual report. London: HM Inspectorate of Prisons, 2017. 
13. Robertson C, Archibald D, Avenell A, et al. Systematic reviews of and integrated report on the quantitative, qualitative and economic evidence base for the management of obesity in men. Health Technol Assess 2014;18:1-424.

14. Public Health England. NHS health check: best practice guidance. London: Public Health England, 2016.

15. Public Health England. Physical health checks in prison webinar: Public Health England, 2016.
16. Kislov R, Walshe K, Harvey G. Managing boundaries in primary care service improvement: a developmental approach to communities of practice. Implement Sci 2012;7:97.

17. Institute for Innovation and Improvement. The Handbook of quality and service improvement tools: NHS England.

18. Royal Society for Public Health. Minded to change: The link between mental wellbeing and healthier lifestyles. London: Royal Society for Public Health, 2016:13. 